\title{
Volume-Rendering on a 3D Hyperwall A Molecular Visualization Platform for Research, Education and Outreach
}

\author{
Preston J. MacDougall ${ }^{1}$, Christopher E. Henze ${ }^{2}$ and Anatoliy Volkov ${ }^{1}$ \\ ${ }^{1}$ Department of Chemistry and Center for Computational Science, Middle Tennessee State University, 1301 E. Main St., \\ Murfreesboro, Tennessee, U.S.A. \\ ${ }^{2}$ NASA Advanced Supercomputing Division, Ames Research Center, Moffett Field, California, U.S.A. \\ \{pmacdougall,avolkov\}@mtsu.edu,chris.henze@nasa.gov
}

\begin{abstract}
Keywords: $\quad$ volume-rendering, chemistry, visualization, STEM outreach, catalysis, biomolecules, drug design
Abstract: We present a unique platform for molecular visualization and design that uses novel subatomic feature detection software in tandem with 3D hyperwall visualization technology. We demonstrate the fleshing-out of pharmacophores in drug molecules, as well as reactive sites in catalysts, focusing on subatomic features. Topological analysis with picometer resolution, in conjunction with interactive volume-rendering of the Laplacian of the electronic charge density, leads to new insight into docking and catalysis. Visual datamining is done efficiently and in parallel using a 4x4 3D hyperwall (a tiled array of 3D monitors driven independently by slave GPUs but displaying high-resolution, synchronized and functionally-related images). The visual texture of images for a wide variety of molecular systems are intuitive to experienced chemists but also appealing to neophytes, making the platform simultaneously useful as a tool for advanced research as well as for pedagogical and STEM education outreach purposes.
\end{abstract}

\section{INTRODUCTION}

Molecular graphics have been an integral part of computational chemistry research since before the advent of electronic computing. Indeed, as chemically- relevant data sets become more and more dense, the need for efficient visual data-mining becomes more and more critical. However, while the speed of number crunching has risen exponentially, the evolution of modes of displaying molecular data has been mostly superficial. Often, the most time-consuming component of graphical processing is ray-tracing for reflecting balls and sticks that garishly represent atoms and bonds. Advances in volume-rendering (Drebin, Carpenter and Hanrahan, 1988) have given depth to molecular images that are essential to capture the "cloudiness" of the quantum world (MacDougall and Henze, 2001).

Chemical understanding and insight often comes from comparison of the properties of a homologous series of molecules. Tiled visual displays are the graphical analogy of tabulated numerical data, and the hyperwall, developed at NASA Ames (Sandstrom, Henze and Levit, 2003), is designed to optimize the efficiency of observing trends and subtle differences in very large and complex, but related, data sets.

This paper presents samples of molecular visualization on a platform that couples volumerendering with hyperwall technology. Applications of this platform have included drug design, catalysis modelling, chemical education, and STEM education outreach.

\section{MOLECULAR AESTHETICS}

Until the invention of Scanning Tunnelling Microscopy, molecules could not be directly visualized. And the recent visualization of a chemical reaction, via non-contact Atomic Force Microscopy (de Oteyza et al., 2013), represents a dramatic premiere in the cinematography of chemical reactions. Nevertheless, all of these images confirm the quantum mechanical condition that it is always cloudy when we look at matter on the nanoscale. Whereas, during the education of a chemist, it is the sharply quantized and fundamental properties of mass and energy that are routinely measured in the laboratory and which prove most useful in the assembly of molecular models that 
form the basis of chemical understanding and discovery. At transition points in the various curricula that students might follow, the visual texture of these models can change drastically and without a clear mapping between models (Figure 1).

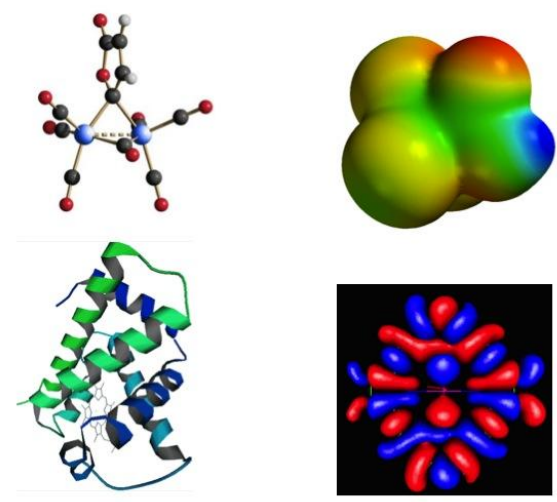

Figure 1: Various types of molecular models commonly used at different stages of a chemical education.

Initially, ball and stick models suffice to convey the concept of molecular structure in General Chemistry. When the characteristic polarities and reactivities of different functional groups need to be depicted in Organic Chemistry, electrostatic potential maps (top-right, Fig. 1) are the norm. The very large structures of biomolecules, such as the proteins and nucleic acids at the heart of Biochemistry, necessitate a simplification in the models using stylized motifs for more-or-less transferable patterns, such as alpha helices (bottomleft, Fig. 1). Atoms, however, are not immutable Lego blocks. They possess subatomic structures that can be virtually indistinguishable, such as the central carbon atoms in pentane and heptane. Or they can have quite distinct topologies, such as the carbon atoms in carbon monoxide vs. carbon dioxide (Bader, 1990). Chemists usually invoke electronic structure models to rationalize the evolution of atomic properties in different molecular environments, and the nature of these models can vary across the periodic table, and even across the hallways of a Chemistry Department. At the subatomic scale, the inherent uncertainty of quantum theory, as well as the many-body nature of atomic physics, precludes any simple depiction of the multidimensional results of computations that are accurate enough to model chemical reactions with high fidelity. But we can't help trying.

As their mathematical skills develop in Physical Chemistry, students will encounter ever more complex models of the electronic structures of molecules; from non-mathematical electron dot diagrams, to so-called molecular orbital calculations that yield multiple images for even the simplest description of a single small molecule. For want of anything that has physical significance, students of Inorganic Chemistry will usually encounter multiple balloon-style images in a pedagogically suspect attempt to depict the allowed and fluctuating electronic states of metals in minerals, enzymes and catalysts, such as the iron atom encapsulated in heme proteins (bottom-right, Fig. 1).

A curriculum-spanning single visual texture, that is faithful to the physical nature of molecules and doesn't create misconceptions, would be pedagogically useful as a supplement to the subdiscipline-specific types of images referred to above. It would help students map one kind of rendering onto the others, and hopefully reduce the large fraction of students that become frustrated along the Chemistry curriculum and change their course of study.

\subsection{Volume-Rendering the Laplacian of the Electronic Charge Density}

The "charge clouds" that are introduced to students at the earliest stage of their chemical education, represent the quantum mechanical probabilities of finding an electron at various points in a molecule. The Laplacian of the total electronic charge density, $\nabla^{2} \rho$, is simply the sum of the second derivatives of the charge density along any three orthogonal axes. It is a scalar function that identifies regions of local concentration $\left(\nabla^{2} \rho<0\right)$ and regions of local charge depletion $\left(\nabla^{2} \rho>0\right)$. The topological properties of this simple, and experimentally measurable, distribution have been shown to provide a physical basis for popular models of chemical reactivity and molecular geometry (Bader, MacDougall and Lau, 1984). Figure 2 demonstrates how volume-rendering can depict key aspects of both the molecular structure (two conjoined rings of bond concentrations in this case), as well as subatomic electronic structures (carbon and nitrogen clearly have two quantum shells of charge concentration), while the terminal hydrogens lack an inner shell. However, some of the hydrogens have local depletions (green yarmulkes), while others do not. This variation is consistent with the propensity of the corresponding hydrogen atoms to form hydrogen bonds, which is one of the most important interactions among biomolecules. 


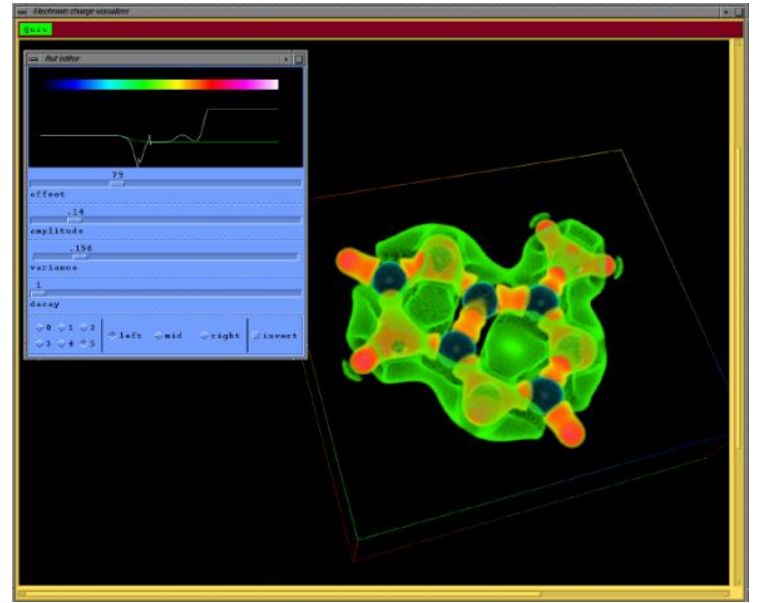

Figure 2: Screenshot of interactively volume-rendering the Laplacian of the computed electron density in adenine. In this and other volume-renderings presented here, warm colours indicate local charge concentration (yellow<orange<red<white), while cool colours represent local charge depletion (green $<$ blue $<$ indigo).

In our implementation of volume-rendering, a tuneable "opacity transfer function" can be adjusted with superimposed Gaussian and step functions, interactively. It has the effect of rendering volume elements in 3D texture memory as opaque, invisible, or with adjustable levels of translucence. The rendering colour is keyed to the value of the Laplacian at that point (Figure 2). Much like dyestained organelles in a cell come into and out of focus under the microscope, topological features that are associated with reactive sites, such as reactive nonbonded electron pairs on the three $\mathrm{V}$-shaped imino nitrogens in an adenine molecule (Figure 2), can be probed for size and shape in threedimensions.

\subsection{Visual Textures of Molecules from Across the Curriculum}

Any molecule, for which one has a high-resolution grid of electron density values (preferably accurate ones), can be rendered in the above manner. This applies to the results of quantum-chemical computations - from low-level approximations that might be part of an Organic Chemistry lab, up to those of spectroscopic accuracy that might be essential for the confirmation of the complex stereochemical structure of a newly discovered natural compound. Additionally, if low-temperature (to reduce molecular vibrations), high-resolution $\mathrm{X}$ ray diffraction data is available for crystalline materials, this same volume-rendering and interactive analysis can be applied to real materials (Coppens and Koritsanszky, 2001).

Organic compounds can be polar (and hence soluble in water, such as fruity esters), as well as nonpolar (and hence insoluble in water, such as hydrocarbons). Drug candidates often have to be hybrids, so that they can enter the digestive system orally, but also be able to cross the oily membranes of cells in order to reach their target. An example is penamecillin, which has an oily fragment (lowerhalf, Figure 3) as well as a water-loving ester-sidechain (upper-half, Figure 3). Like all penicillin derivatives, it has an electron-rich sulphur atom (central orange lobes, marked with arrows in Figure 3) adjacent to a strained nitrogen-containing lactam ring. To the left of the sulphur atom, a green yarmulke is marked with an arrow on the only hydrogen (out of 22) that conventional rules assign as potentially hydrogen bonding. This central pocket is the active site of the drug.

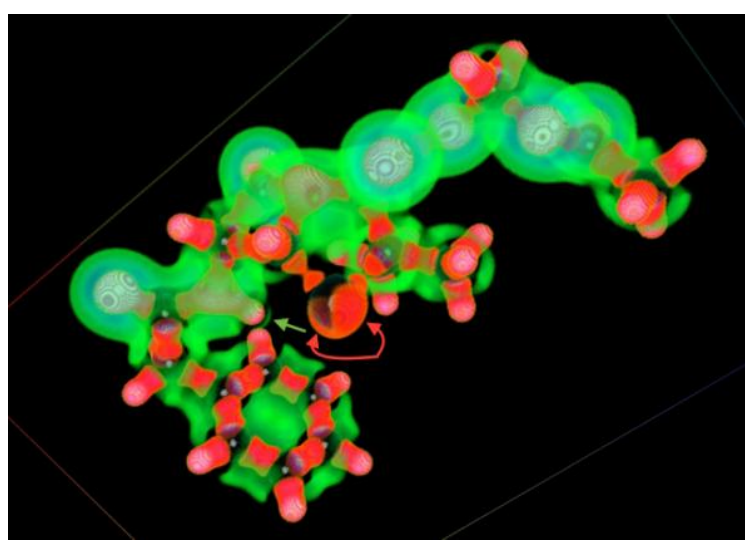

Figure 3: Volume-rendering of $\nabla^{2} \rho$ for penamecillin.

It is important to note that the application of conventional rules, in order to attempt to predict the activity of drug candidates, requires knowledge of atomic identities and usually that of their nearest neighbours. For instance, conventional hydrogen bonds can be formed only by a hydrogen atom that is bonded to nitrogen, oxygen or fluorine. Whereas the volume-rendering of $\nabla^{2} \rho$, and the chemical interpretations based on it, are blind to atomic identity and conventional rules. (A grid of probability values is the only data input.) Molecules do not respond to rules and conventions. They respond to the physical forces that, especially at close range, are determined by the lumps and holes in the charge distributions of molecules. The topological properties of $\nabla^{2} \rho$ define a molecule's 
physical texture, and its volume-rendering of this data gives it a corresponding visual texture.

Becoming familiar with the visual textures of different kinds of chemical moieties will require extensive experience on the part of the student, but that is true of learning any computational chemistry platform. In the present case, the experience is fun because the manipulated visualizations are of physical data, and not simply the virtual equivalent of a plastic molecular modelling kit, with arbitrary balls and sticks.

Organometallic compounds are those that contain organic groups as well as metals atoms, often transition metals such as iron. The active sites in enzymes, especially those involved in reduction and oxidation processes, are often organometallic in nature. In addition to the extreme difficulties that such compounds present with regard to modelling their very complex electronic structure, visualizing the resulting data offers unique challenges on its own. Transition metals are characterized by their dual possession of loosely-held valence electrons that give rise to high electrical conductivity and the formation of metal-metal bonds, but also tightly-held inner-shell electrons that can give rise to magnetic properties and unusual stereochemical behaviour not seen in organic compounds. The Laplacian of the electron density differs by several orders of magnitude at critical points in the topological features associated with these split characteristics. And the topological features associated with the organic moieties are somewhere in between. Interactive volume-rendering is able to simultaneously capture the visual textures associated with these disparate molecular environments, and also characterize their evolution during their signature catalytic processes.

As an example, certain bacteria can reduce the nitrogen in the atmosphere to form ammonia, which is then used to synthesize the building blocks of proteins and DNA needed by all living organisms. The enzymes enabling such "nitrogen fixation" belong to the nitrogenase group. All group members possess iron-sulphur clusters at their active sites, and chemists are very interested in building synthetic mimics of these catalysts for energy-efficient means of producing valuable and high-energy nitrogencontaining compounds out of thin air.

Figure 4 is a montage of images for a synthetic analogue of an active site of nitrogenase enzymes. It efficiently reduces diazene $\left(\mathrm{N}_{2} \mathrm{H}_{2}\right)$ to ammonia $\left(\mathrm{NH}_{3}\right)$, which is an essential intermediate step in nitrogen fixation. Again, a single volume-rendering can highlight details of the covalent bonds (reddish features) and molecular structure that holds this assembly in just the right position. And at the same time, key topological features near the core of the iron atoms, that are associated with the electrochemical activity of the system, are wellresolved and can be individually characterized. Evolution of this intricate topology during the reduction process will lead to a greater understanding, and hopefully catalyst refinement.

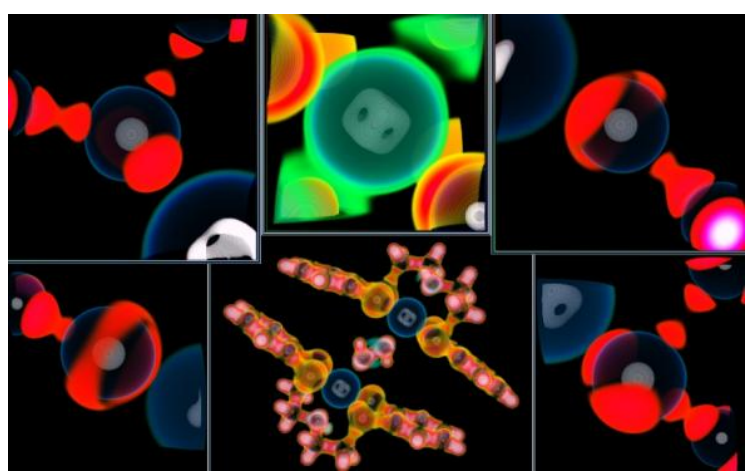

Figure 4: Volume-rendering of $\nabla^{2} \rho$ for diazene (at centre of the lower-middle image) bound to a di-iron cluster encapsulated by organosulphur ligands (corners). The pair of iron atoms are the bluish balls in the lower-middle image, one of which is shown up-close in the uppermiddle image.

The catalytic activity of inorganic catalysts is an inherent property of the often-complex molecular and electronic structures of the catalyst. However, in addition to structural properties of the reactive site, the catalytic activities of large biomolecules are also marshalled by large-amplitude motions of their key structural components, such as gates and channels. In the co-enzyme vitamin $\mathrm{B}_{12}$, secondary access to the cobalt atom at the active site is controlled by an adenine-like group that swings open and shut in a gate-like motion. Doors and gates require hinges, and in Video 1 below (URL cited in references; Kemp, 2001), the rendering of the phosphate group (lower right), that functions as the molecular hinge, resembles a group of ball-bearings that can freely roll around one another. This is in contrast to the rest of the molecule, which has a more rigid-looking structure of directed covalent bonds and their local concentrations of electronic charge that are rendered orange-red.

This example illustrates that the visual texture that is revealed by interactive volume-rendering of $\nabla^{2} \rho$ reveals information that is tied to the physical dynamics of complex molecular systems, as well as their chemical characteristics. Such visualizations during biochemical processes could help sort-out the 
poorly understood role of the interplay between large-amplitude motions of biomolecules and their chemical reactions.

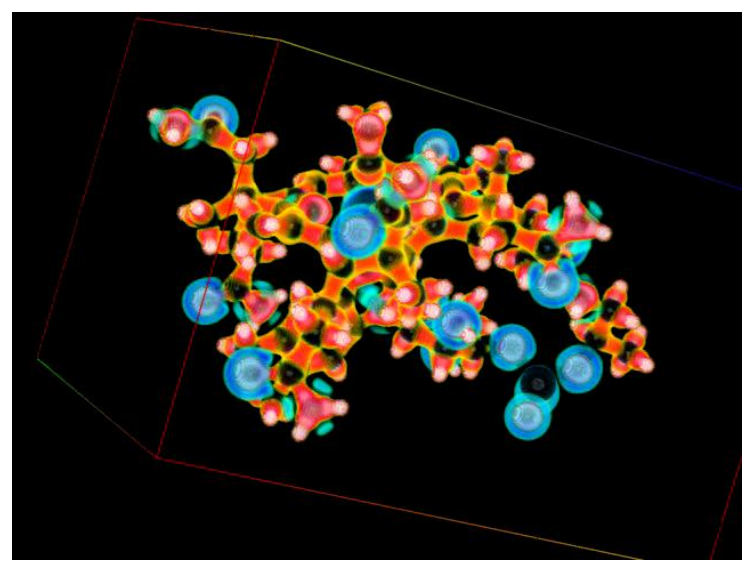

Video 1 . Volume-rendered $\nabla^{2} \rho$ for vitamin $\mathrm{B}_{12}$ pirouette.

\subsection{Molecular Visualization on a 3D Hyperwall}

Like enzymes, many drugs belong to families of chemically related compounds. Penamecillin is a member of the large class of penicillins. The differences between structures can be small, while the pharmacological differences can be large. Just as with trying to discriminate between physical objects that are similar but not the same (such as a blind taste test), side-by-side comparisons are indispensible. Drug molecules are dynamic objects, and their different responses to identical stimuli can be just as important to differences in activity as are any structural differences. Hyperwall technology (Sandstom, Henze and Levit, 2003) provides unique capabilities for understanding the similarities and differences between large sets of data, such as charge density data for a series of related drug molecules (MacDougall and Henze, 2007). A simple tiled array of high-resolution 3D molecules can provide parallel comparisons, but the synchronized and real-time sharing of functional information between rows (or columns) on a hyperwall allows much more than a superficial comparison.

We have illustrated the effectiveness of volumerendering of $\nabla^{2} \rho$ for molecules of various composition. Key to its effectiveness, although not easily shown in print, is the interactivity of the process of "focusing" on different topological features via the opacity transfer function. When the same scanning is done simultaneously on related molecules, side-by-side volumetric comparisons are done, as opposed to purely superficial ones. Even the slightest difference, completely obscured in most surface renderings, will jump out at you - especially in $3 \mathrm{D}$ !

\subsection{Chemical Education and STEM Outreach with a 3D Hyperwall}

The 3D hyperwall at MTSU was completed in 2012, and research in drug design is ongoing. Concurrently, three inter-disciplinary science $\mathrm{PhD}$ programs were initiated at MTSU in 2010 (the university's first in the sciences). One is in Computational Science, and graduate students from diverse educational backgrounds are beginning to experiment with new ways to use the hyperwall in their research (not just molecular). Another new $\mathrm{PhD}$ program is in Mathematics and Science Education. Future pedagogical research is planned on the effectiveness of volume-rendering of $\nabla^{2} \rho$ as an alternative to the standard molecular modelling labs in Organic Chemistry courses. The same could be done in other chemistry subdisciplines, such as Inorganic Chemistry and Biochemistry, although molecular modelling labs are not commonplace in these subjects. Perhaps they will be in the future, if our educational research and software development is successful.

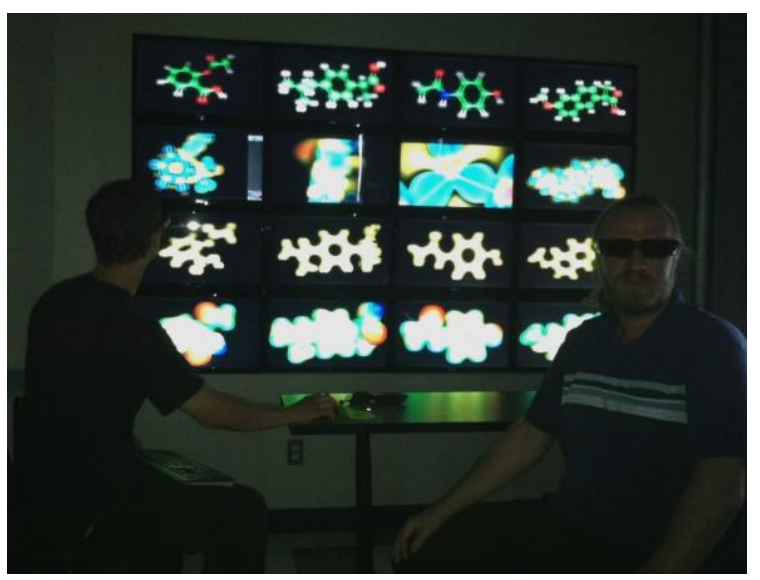

Figure 5: The 3D hyperwall at MTSU, with various renderings of a series of related drug molecules. Dr. Anatoliy Volkov (right) supervises as Mr. Chris Irwin (a Chemistry student researcher) engages in drug discovery.

The important aspect of this proposed research is the consistent visual texture that would be used across the curriculum. In this way, advances in understanding one subject will spill over to other subjects. Currently, because of the disparate visual 
textures used for different subdisciplines, students tend to underestimate the overlap of the subjects.

Stimulating and insightful molecular graphics can enhance chemical education, but only if the students are signing-up for the courses. There are concerted efforts on the part federal and state Departments of Education in the U.S.A., in conjunction with Chambers of Commerce from around the country, to increase the numbers of college-bound students that are interested in pursuing studies in STEM disciplines (science, technology, engineering and mathematics). Many beginning students at MTSU (and other large public universities) express interest in molecular science, but lack the mathematic preparation to succeed (without doing at least one year of mathematics that should have been done in high-school). To address this problem, many university researchers are actively involved in outreach activities that expose high-school students, and younger, to the excitement of science and scientific research. These activities are hands-on whenever possible, and the take-home message invariably includes the importance of taking as many mathematics classes as possible while still in high-school.

For the last two years, two of us (PJM and AV) have conducted a 3D Drug Design Workshop for high-school girls (15+ participants). This introduces participants to the nature of computational science, and drug design software in particular. They learn a little about how software is written, and that it is essentially applied mathematics. They see colourful volume-renderings of organic molecules, which they invariably describe as "Beautiful!" (Kemp, 2001). They learn how the size, shape and electrical properties of drug molecules determines their "fit" to biological targets. Although the girls usually only know the little bit of organic chemistry that they have been exposed to in Biology class (or picked-up from TV shows like "Breaking Bad"), the girls have no trouble making suggestions to modify existing drugs in a competition to see which group can get the highest docking score.

In addition to learning about computational science and drug design, the girls learn that computer graphics is not just for games - although it can be fun (Mahoney, 2001). Comments received by the conference organizers included gems like "I loved the 3D modeling workshop! I had heard horror stories about Organic Chemistry, but now I can't wait to take it!"

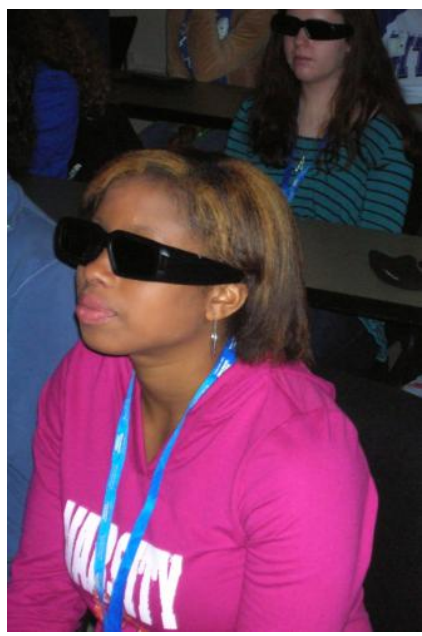

Figure 6: A high-school student participating in the 3D Drug Design Workshop of the 2012 Expanding Your Horizons Conference at MTSU.

\section{CONCLUSIONS}

With innovative use of rendering algorithms, in tandem with the functionally interconnected display capabilities of a hyperwall, molecular visualization research can not only help us see the data that molecular simulation generates - at ever increasing speeds - but it can be the key to gaining understanding from it.

And, since understanding is not just the key to discovery in research, but also the key to discovery in learning, volume-rendering of $\nabla^{2} \rho$ (not necessarily on a hyperwall, although that would amplify understanding) can be a powerful pedagogical tool for chemical education.

Finally, in the long-term, advances in scientific research, along with the economic stimulus that accompanies it, will depend on recruiting the most imaginative and energetic students into STEM disciplines. Visually exciting computational modelling, as a complement to laboratory-based research, is a fun and effective way to learn chemistry, but it might become essential to attract, retain and inspire today's increasingly visuallyoriented youth. The interactivity of the software, combined with the wow-factor of the 3D-hyperwall, give this modelling technology a game-like feeling, complete with the search and discovery of topological features. This naturally leads to the opportunity of porting the technology to a single workstation for the development of education games. 


\section{ACKNOWLEDGMENTS}

Financial support at MTSU is acknowledged from the Office of Science, U.S. Department of Energy (DE-SC0005094).

\section{REFERENCES}

Bader, R. F. W., 1990. Atoms in Molecules: A Quantum Theory, Clarendon Press, Oxford.

Bader, R. F. W., MacDougall, P. J., Lau, C. D. H., 1984. J. Amer. Chem. Soc., 106, 1594-1605.

Coppens, P., Koritsanszky, T., 2001. Chemical Reviews, 101, 1583-1627 (and references therein).

de Oteyza, D. G., Gorman, P., Chen, Y.-C., Wickenburg, S., Riss, A., Mowbray, D. J., Etkin, G., Pedramrazi, Z., Tsai, H.-Z., Rubio, A., Crommie, M. F., Fischer, F. R., 2013. Science, 340, 1434-1437.

Drebin, R. A., Carpenter, L., Hanrahan, P., 1988. "Volume rendering" in Computer Graphics (Ed.: J. Dill), SIGGRAPH '88 Proceedings, 22, 65-74.

Kemp, M., 2001. Nature, 412, 588. (The mpeg file that is discussed in this article, can be downloaded from: http://www.mtsu.edu/honors/images/B12.mpg)

MacDougall, P. J., Henze, C. E., 2001. Theor. Chem. Acc., 105, 345-353.

MacDougall, P. J., Henze, C. E., 2007. "Fleshing-out Pharmacophores with Volume-Rendering of the Laplacian of the Charge Density and hyperwall Visualization Technology", chapter 19 in "The Quantum Theory of Atoms in Molecules: From Solid State to DNA and Drug Design" C. F. Matta and R. J. Boyd (eds.), Wiley-VCH, pp. 499-514.

Mahoney, D. P., 2001. "Atomic Vision" Computer Graphics World, 24, 15.

Sandstrom, T. A., Henze, C. E., Levit, C., 2003. "The hyperwall", in Coordinated and Multiple Views in Exploratory Visualization, IEEE Conference Proceedings, 124-133. 


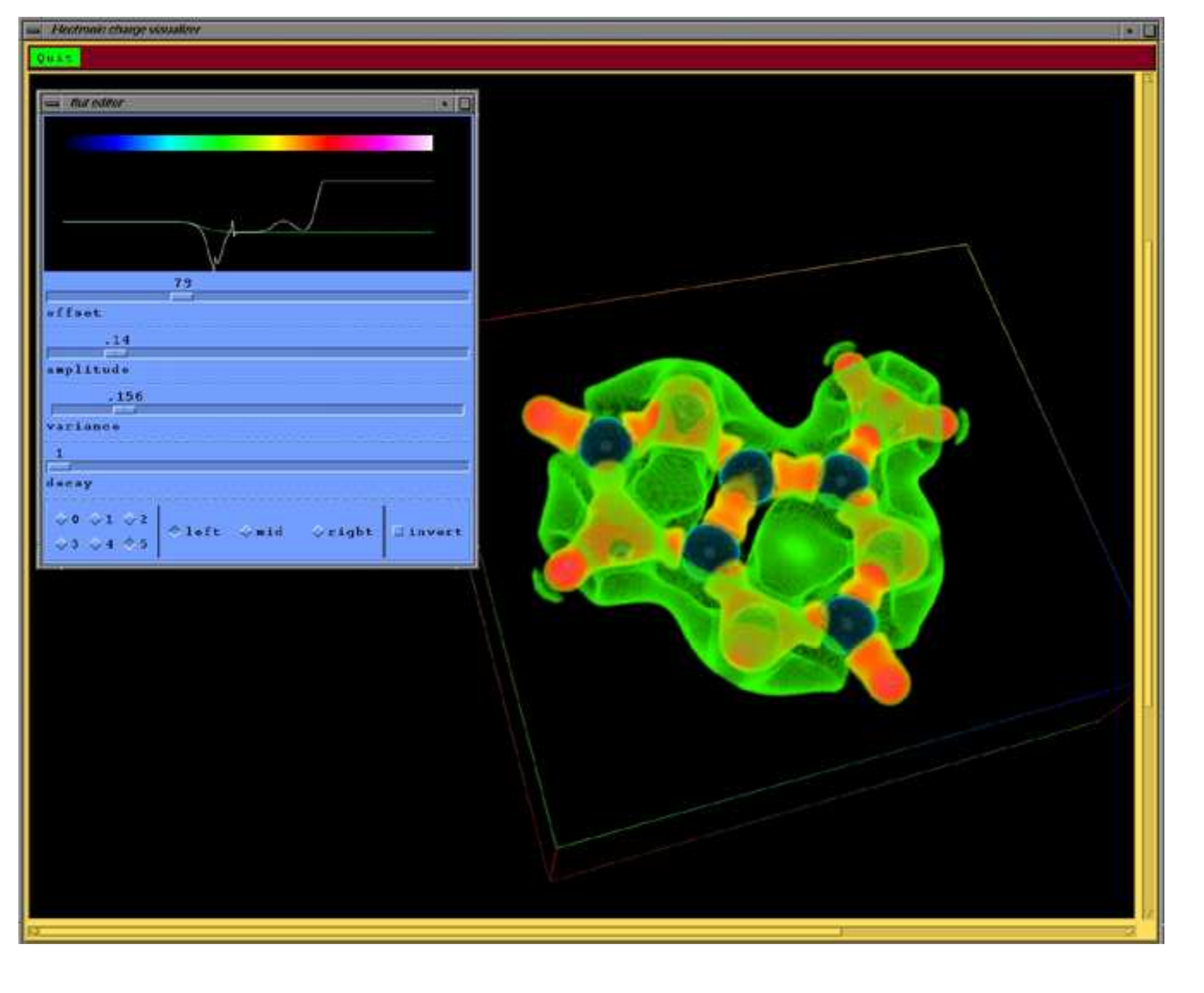

. 